\title{
Run-to-Run Sequencing Variation Can Introduce Taxon-Specific Bias in the Evaluation of Fungal Microbiomes
}

\author{
Zewei Song ${ }^{\dagger}$ and Dan Schlatter, Department of Plant Pathology, University of Minnesota, Saint Paul; Daryl \\ M. Gohl, University of Minnesota Genomics Center, Minneapolis; and Linda L. Kinkel, Department of Plant \\ Pathology, University of Minnesota, Saint Paul
}

Accepted for publication 6 July 2018.

\begin{tabular}{|c|c|}
\hline $\begin{array}{l}\text { The routine use of high-throughput sequencing to profile microbial } \\
\text { communities necessitates improved protocols for detecting and } \\
\text { adjusting for variation among sequencing runs for marker gene } \\
\text { analysis. Although mock communities are widely used as a control } \\
\text { among runs, the composition and diversity of mock communities, in } \\
\text { most cases, are orders of magnitude lower than the actual samples. } \\
\text { We demonstrated that replicated biological samples ("technical } \\
\text { replicates") are superior to a mock community in detecting variation } \\
\text { and potential bias among sequencing runs. We present a case in }\end{array}$ & $\begin{array}{l}\text { which technical replicates of three soil samples were sequenced } \\
\text { in three MiSeq runs containing samples from multiple experiments. } \\
\text { The technical replicate samples revealed a potentially biased, outlier } \\
\text { sequencing run, from which several Ascomycota taxa were } \\
\text { substantially underestimated. Similar bias was seen in the other } \\
\text { samples sequenced but was not detected using the mock } \\
\text { community. Our study demonstrates that using technical replicates } \\
\text { along with traditional mock communities provide additional quality } \\
\text { control information and aid in detecting outlier sequencing runs. }\end{array}$ \\
\hline
\end{tabular}

The detailed study of plant microbiomes is fundamentally changing our understanding of phytobiomes. With advances in high-throughput sequencing (HTS) techniques, the composition of microbial communities from large numbers of samples can now be analyzed at low cost and great depth (Caporaso et al. 2012; Yatsunenko et al. 2012). As an emerging technique, researchers continue to assess the reliability of sequencing technologies to accurately reflect the composition and diversity of microbial communities within the phytobiome (Bokulich et al. 2013; Cole et al.

${ }^{\dagger}$ Corresponding author: Z. Song; E-mail: songzewei@outlook.com

D. M. Gohl is a shareholder and CSO of CoreBiome, Inc.

Author contribution: Z.S., D.S., D.M.G., and L.K. wrote the main manuscript text. Z.S. prepared the figures and tables. Z.S. and D.S. collected soil samples and extracted DNA. D.M.G. provided support on the sequencing. All authors reviewed the manuscript.

Current address of D. Schlatter: USDA-ARS Wheat Health, Genetics, and Quality Unit, Washington State University, Pullman.

Funding: This project was supported by University of Minnesota MnDRIVE project funds, Minnesota Agricultural Experiment Station Project MIN-22-018, and NSF Macrosystems Award EF-124189.

*The $\boldsymbol{e}$-Xtra logo stands for "electronic extra" and indicates that six supplementary figures and four supplementary files are published online.

(C) 2018 The American Phytopathological Society
2009; Schloss et al. 2011). However, we are still far from standardized protocols for controlling the quality of amplicon sequencing data. Several major sources of error can contribute to biased characterization in HTS of microbial communities. Sample collection and storage conditions can affect the stability of the community (Penton et al. 2016). In addition, differences in the efficiency of DNA extraction for different taxa can introduce bias (Costea et al. 2017). Errors introduced during PCR amplification can also skew observed species composition due to primer mismatches, amplicon length variation, or PCR conditions (e.g., annealing temperatures and cycle numbers). These errors are well studied and can be reduced by (i) using improved primer sets that amplify to a wider range of microbial groups (Klindworth et al. 2013), and (ii) constraining PCR cycle number, optimizing template concentration, and using highfidelity polymerase (Gohl et al. 2016; Lindahl et al. 2013). Current bioinformatics tools can also mitigate PCR-based errors through chimera identification (Edgar et al. 2011; Haas et al. 2011).

There are currently no standards or best practices for quality control in combining amplicon sequencing data across multiple runs. For many studies in which only one sequencing run is used, inter-run variability is not a concern. However, as the cost of sequencing continues to decline, sample sizes are likely to routinely exceed the capacity of a single run for many studies. Depending on sample type and desired read depth, one might typically run 96 to 384 samples in a MiSeq $300 \mathrm{PE}$ or HiSeq $250 \mathrm{PE}$ run. Thus, projects with thousands of samples or samples that are processed at different times will require the use of multiple sequencing lanes. By default, 
randomization should be used to minimize batch effects, but studies that evaluate variation and batch effects due to sequencing run are surprisingly scarce. Two recent studies compared variation among different sequencing machines, but not among runs on the same type of machine, which is the more common case for real studies (Caporaso et al. 2012; D'Amore et al. 2016).

Here we examine the variation introduced by multiple sequencing runs. We randomly selected three soil samples to use as technical replicates. For each selected sample, three independent PCR libraries were prepared targeting the fungal ITS1 region, and each was sequenced three times in each of three MiSeq runs. A single replicate of a 27-species mock community used in Nguyen et al. (2015) was also included in each run. Upon observing a significant run effect on our sequencing data, the sources of run variation and potential solutions to reduce the run effect were explored. Our results suggest that routine inclusion of technical replicates in amplicon sequencing runs should be considered as a best practice for reducing the impacts of run variation on study results.

\section{MATERIALS AND METHODS}

HTS. For clarification, we use the term "replicated sample" to refer to the soil samples $(n=3)$ that were selected to repeat in each run. We use the term "technical replicate" to refer to the individual samples repeatedly sequenced within each run $(n=3$ technical replicates for each of the three replicated samples in each run). Thus, the total number of technical replicates for each replicated sample is nine across three runs.

For evaluating the reproducibility and variation of different sequencing runs, we randomly picked three soil samples (soil samples 110-1, 138-2A, and 182-2) and sequenced them along with samples from two separated experiments, across three runs (Fig. 1). Each replicated soil sample was sequenced three times in each of three individual runs (run 1, run 2, and run 3) for the fungal ITS1 region, generating a total of nine technical replicates for each soil. The three soil samples were randomly selected from a collection of soil samples from a plant diversity-controlled experiment at the Cedar Creek Ecosystem Science Reserve (CCESR), a National Science Foundation Long-Term Ecological Research site in east-central Minnesota (http:// www.cedarcreek.umn.edu/). The diversity treatment consists of 84 samples from monoculture plots, and 88 samples from 16-species polyculture plots (Essarioui et al. 2017). Besides the nine technical replicates for the three different soil samples, sequencing run 1 contained 88 soil samples from two potato fields (Becker and Rosemount, $\mathrm{MN}$ ) representing distinct agricultural habitats (referred to as experiment I in Fig. 1). The 172 CCESR samples were randomly allocated into run 2 and run 3 (referred to as experiment II in Fig. 1). Finally, each run also contained a fungal mock sample (27 fungal species represented by equal amounts of DNA) (Nguyen et al. 2015).

The sequencing libraries were prepared following Smith and Peay (2014). For each replicated sample, we prepared three pooled PCR libraries, and each was a mixture of three PCR reactions with annealing temperatures at 50,53 , and $55^{\circ} \mathrm{C}$ (Fig. 1). All PCR mixtures were purified and pooled in equal molar ratios and sequenced in the University of Minnesota Genomic Center on three separate MiSeq $2 \times 250$ bp runs. Thus, for each sample serving as a technical replicate, we prepared three separate PCR mixtures, and sequenced these three PCR mixtures in three runs for a total of nine technical replicates. This allowed us to distinguish sequencing variation due to PCR and variation due to sequencing run. A blank sample following the same extraction procedure was used as an extraction control for each library.

Data processing. Raw data were processed using our customized Python pipeline (FAST, https://github.com/ZeweiSong/FAST).
Following the suggestion by Nguyen et al. (2015) for fungal amplicon data, we used Read1 sequences for all analyses. All sequences were labeled with sample name (for example, SampleA_121). Sequencing primers were removed from all sequences, along with a highly-similar 26-bp head which belongs to 18S. Any sequence with expected error rate $>1$ was discarded using VSEARCH (Rognes et al. 2016). Any sequence with homopolymers longer than nine (not including nine) and ambiguous bases were also discarded. The quality filtered sequence file was deprelicated. Singletons in the dereplicated file were discarded. We then used VSEARCH to perform chimera checking and operational taxonomic unit (OTU) clustering. Potential chimeras were picked against the UNITE database (version 7.2 with singletons set as RefS) with the "uchime_ref" function in VSEARCH. OTUs were picked and used "cluster_size" function, in which sequences were sorted by the size of dereplicated units, with a similarity of 0.97 . The VSEARCH output was parsed according to the format of FAST. An unrarefied OTU table was then generated along with the representative sequences for all OTUs. The abundance of OTUs in extract controls (one for each run) were subtracted from their corresponding samples. We assigned taxonomy to OTUs using VSEARCH against the UNITE database plus the sequences of the

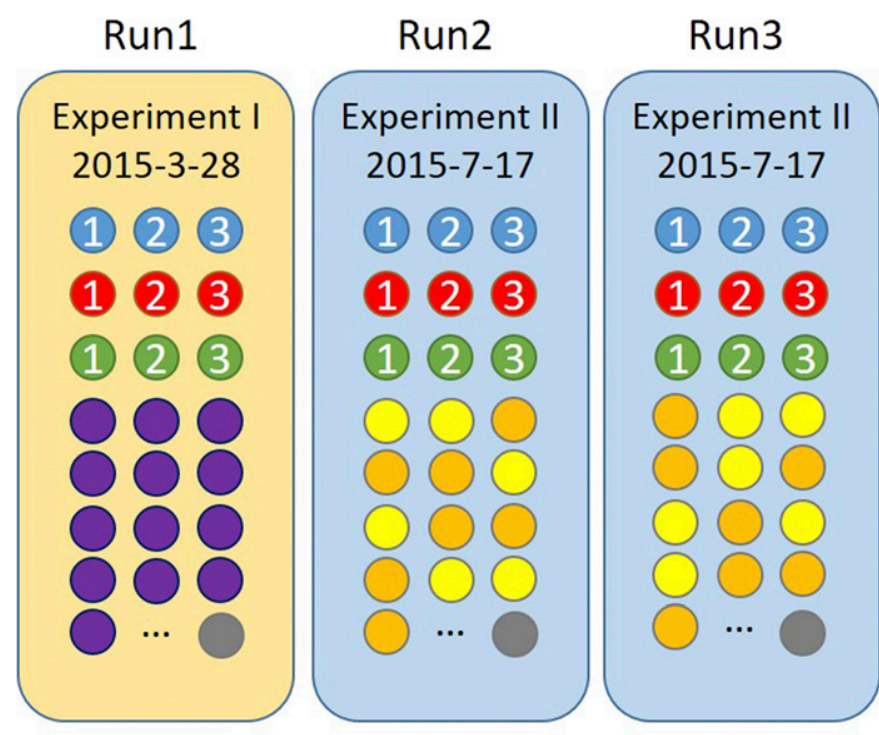

$110-1$

$138-2 \mathrm{~A}$

\section{$182-2$}

\section{Samples from Experiment I} Samples from Experiment II Mock community

Fig. 1. The allocation of samples in the three sequencing runs. The blue, red, and green circles indicate the three samples used as technical replicates in each run. Numbers inside each circle indicate PCR library. Three PCR reactions for fungal ITS1 were combined, and the resulting three libraries was sequenced across the three runs. The date inside each run box indicates when data were released by the sequencing center. Additional biological samples from the agricultural experiment were sequenced in run 1, while samples from the Cedar Creek Ecosystem Science Reserve plant diversity treatment were randomly allocated between run 2 and run 3 . 
27 mock species. Any OTU with similarity (Pident) $>75 \%$ over $>70 \%$ sequence length (Match\%) is considered to belong to the fungal kingdom, all other OTUs were discarded.

Evaluation of run effect. All samples were rarefied to the depth of 19,000 (with 1,000 iterations for each sample) using FAST. The effect of run variation on microbiome composition was tested using both the replicated samples and the samples from CCESR experiment (experiment II sequenced in run 2 and run 3 ). For each replicated sample, we tested the sample similarities among either run or PCR using the ANOSIM function in the vegan package in R (Oksanen et al. 2017). Our expectation is that ANOSIM's R for the PCR effect will be close to 1, and ANOSIM's R for run effect will be 0 (i.e., the only variation we observed is from different PCR libraries, but not different sequencing runs). For all samples from the CCESR experiment (after excluding the three replicated samples), we tested sample similarities among either run or diversity treatments using ANOSIM. Our expectation is that we will observe significant effects of plant diversity treatment but not sequencing run on microbiome composition. Bray-Curtis dissimilarity matrices calculated based on Hellinger-transformed data were used. Nonmetric multidimensional scaling (NMDS) plots were generated using the metaMDS function (with $\mathrm{k}=2$, and trymax $=100$ ) in the vegan package (Oksanen et al. 2017) in $\mathrm{R}$ version 3.4.2 (R Core Team 2017).

Identification of variable OTUs. Upon observing that run 2 was significantly different from run 1 and run 3, we identified OTUs that varied significantly in abundance among runs, thus contributing to the run variation. These variable OTUs (vOTUs) were identified from our data using the following criteria: (i) the OTU is among the top $99 \%$ of total sequences in the rarefied and ranked abundance OTU table, and 2) the average abundance of this OTU among all three technical replicates for a replicate soil sample in run 2 is $<5 \%$ of the average abundance for all nine technical replicates for that soil sample across the three runs (an example working sheet is provided in the supplementary material). Resulting vOTUs across the three replicate soil samples were removed from the original unrarefied OTU table for all samples, and then the OTU tables were rarefied again to 15,000. Run effects were evaluated again with the revised dataset using ANOSIM.

Influence of data processing. In order to evaluate the potential for the data processing to influence sensitivity in detecting run variation, we also processed the sequences using various quality filtering thresholds and clustering methods. After labeling all sequence files and merging them into a single FASTQ file, we processed the data using all possible combinations of the following four parameters in order: (i) read length: sequences were either truncated to a fixed length of $180 \mathrm{bp}$ (sequences shorter than $180 \mathrm{bp}$ were discarded), or leave as it is; (ii) maximum expected error (maxEE): sequence was discarded if its maximum expected error is larger than 1 or 5; (iii) clustering method: we used VSEARCH for de novo clustering, and NINJA-OPS for closed-reference clustering (Al-Ghalith et al. 2016); and (iv) clustering similarity at $0.97,0.90$, and 0.80 for both clustering methods. This resulted in a total of 24 datasets, i.e., OTU tables $(2 \times 2 \times 2 \times 3=24)$, including the one used in above analyses.

All 24 OTU tables resulting from different data processing pipelines were rarefied (with 1,000 iterations for each sample) to a single depth of 10,000 for comparison. Again, ANOSIM was used to evaluate the effect of PCR and run on microbiome composition for the three replicated samples, and the effect of treatment and run for experiment II samples. We also identified and removed all vOTUs from these data using the above criteria and evaluated run effects using the same method. For the vOTUs identified from these 24 datasets, we compared their ITS 1 length and GC content with all the other OTUs in the corresponding OTU table. The ITS1 length and GC content were calculated by identifying the ITS1 region in UNITE database using ITSx (Bengtsson-Palme et al. 2013).

Data availability. The raw sequencing data were deposited to the Data Repository for the University of Minnesota (DRUM, https://conservancy.umn.edu/). The accession number for experiments I and II are 190418 and 190417, respectively.

\section{RESULTS AND DISCUSSION}

Among three runs that were sequenced at different times and on three different MiSeq instruments, we did not observe significant differences in the Read1 quality profile and other sequencing parameters among runs (Supplementary File S1).

The mock communities were virtually indistinguishable among runs (Supplementary Fig. S1, also see the OTU table in Supplementary File S2). Twenty-three fungal species, out of a total of 27, were recovered in all three runs, all with highly similar relative abundance. These results are consistent with other studies that used this mock community (Nguyen et al. 2015). Wilcoxina mikolae was not detected in run 2 and was present at extremely low abundance in both run 1 and run 3 .

When considering the technical replicates, the data show significant variation across sequencing runs, with run 2 differing significantly from the other two runs. Technical replicates sequenced in run 2 were differentiated from those in run 1 and run 3 on NMDS plots (Supplementary Fig. S2), and a significant run effect was detected based on ANOSIM (Table 1, ANOSIM). A similar run effect was observed for the 172 samples from the monoculture and 16-species plots, which were randomly allocated to run 2 or run 3 (Fig. 2A). Specifically, samples were differentiated based upon diversity treatment (monocultures versus 16-species plant communities) along NMDS1, while run 2 and run 3 samples were differentiated along NMDS2.

By manually examining the nine technical replicates for each replicated soil sample, we found that some OTUs had significantly lower abundance in run 2 than in run 1 and run 3 . Using the criteria

TABLE 1

ANOSIM analysis of the run and PCR effect in original and fixed operational taxonomic unit (OTU) table ${ }^{a}$

\begin{tabular}{|c|c|c|}
\hline Sample & Run & PCR \\
\hline \multicolumn{3}{|l|}{$110-1$} \\
\hline Original & 0.358 & $0.671^{\star \star}$ \\
\hline Fixed & 0.268 & $1.000^{\star *}$ \\
\hline \multicolumn{3}{|l|}{$138-2 A$} \\
\hline Original & $0.679^{* *}$ & 0.309 \\
\hline Fixed & 0.309 & $1.000^{\star \star \star}$ \\
\hline \multicolumn{3}{|l|}{$182-2$} \\
\hline Original & $0.465^{\star}$ & $0.399^{*}$ \\
\hline Fixed & $0.309^{*}$ & $1.000^{\star *}$ \\
\hline \multicolumn{3}{|c|}{ Cedar Creek all other samples } \\
\hline Original & $0.421^{\star \star \star}$ & $0.590^{\star \star \star}$ \\
\hline Fixed & $0.209^{* * *}$ & $0.629^{\star \star \star}$ \\
\hline \multicolumn{3}{|c|}{ Cedar Creek all samples } \\
\hline Original & $0.388^{\star \star \star}$ & $0.621^{\star \star \star}$ \\
\hline Fixed & $0.198^{\star \star \star}$ & $0.654^{\star \star *}$ \\
\hline
\end{tabular}


described in the methods, 127 out of 6,555 OTUs (0.3\%) were identified as variable (vOTUs) among the nine technical replicates across the three runs (38 OTUs from 110-1, 71 from 138-2A, and 58 from 182-2, Supplementary File $\mathrm{S} 3$ provides details). The vOTUs were dominated by classes of Ascomycota, among which Sordariomyceters and Dothideomycetes were the most abundant, followed by Archaeorhizomycetes, Eurotiomycetes, and Leotiomycetes (Fig. 2C, Supplementary Fig. S3) in our technical replicates. Notably, vOTUs identified in our study represent functionally important taxa in soil systems, suggesting that these errors may significantly influence biological understanding of the microbiome. For example, several vOTUs belong to Fusarium spp., which includes important plant pathogens as well as populations that play important roles in disease suppression and nutrient cycling in soil (Essarioui et al. 2017).

The abundance of vOTUs identified using technical replicates were also generally lower in run 2 than run 3 for the other samples in experiment II. Among the 127 vOTUs, only eight ( $\sim 6 \%)$ have higher abundance (average among all nonreplicated samples) in run 2 than that in run 3 (Supplementary Fig. S4). Several vOTUs identified in one replicated soil sample were less variable in other replicated samples in the same run. For example, OTU_10 was a rare OTU in run 2 but not run 1 or run 3 for sample 182-2, and was thus assigned as a vOTU, but was equally abundant in all runs for samples 110-1 and 138-2A. This illustrates the limitations of using technical replicates for evaluating the run effects and suggests that a mixture of biological samples could be a better alternative for detecting sequencing errors or bias.

Although we were able to detect a biased run using technical replicates, the approach for correcting such bias needs further investigation. Removing the detected vOTU significantly reduced run effect (Fig. 2B), though still significant (from 0.42 to 0.21 in ANOSIM, $P<0.001$ for both analyses). In contrast, following removal of the vOTUs, the treatment effect increased slightly (from 0.59 to $0.63, P<0.001$ for both analyses) for the 172 samples in run 2 and run 3 (Table 1). However, it should be emphasized that removing OTUs could potentially alter the structure of the compositional data (Gloor et al. 2017). In our study, we tried to minimize such influence by removing vOTUs from the unrarefied OTU table (as described in the Methods). A statistically rigorous approach for
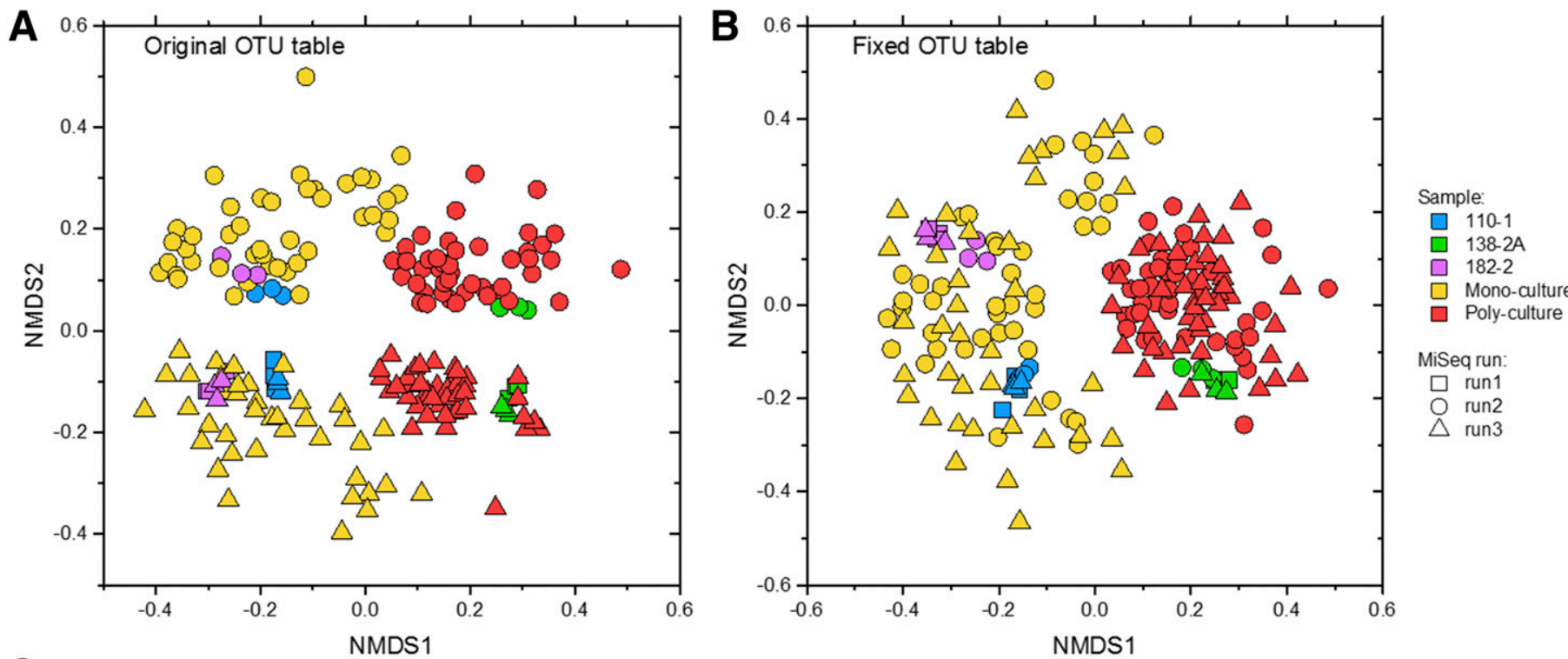

MiSeq run:

$\square$ run1

$\bigcirc$ run2

$\triangle$ run3

C

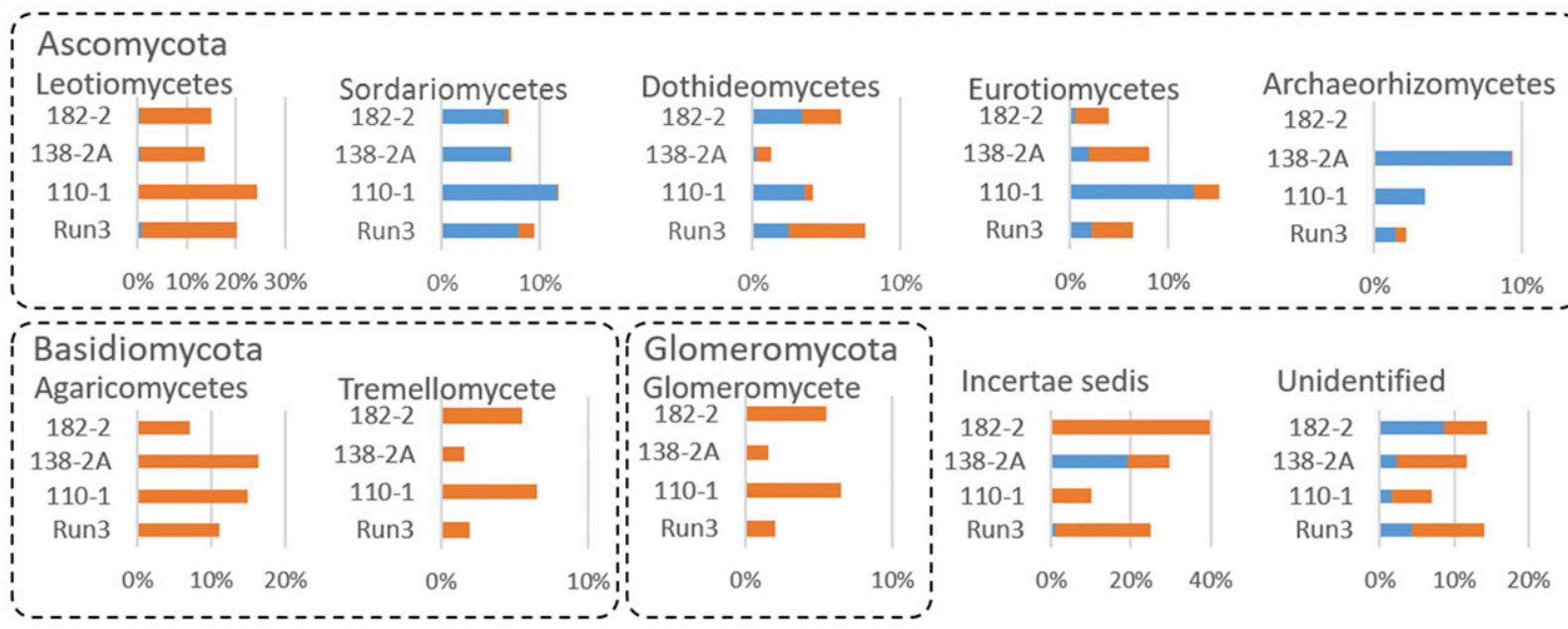

Fig. 2. Illustration of the detection and reduction of run effects, and the proportion of variable operational taxonomic units (vOTUs) in run 3 fungal class by abundance. Non-metric multidimensional scaling (NMDS) plots illustrate A, run effects using the original operational taxonomic unit (OTU) table, and B, the reduction of run effects after removal of variable OTUs (vOTUs). C, The relative abundance of vOTUs (blue bars) versus other OTUs (orange bars) in different fungal classes in run 3 are shown for the three technical replicates and all other samples (labeled as run 3 ). 
any data "corrections" based on vOTUs will be critical for the full utilization of technical replicates in the future.

Increasing error tolerance in data processing did not help to reduce the observed run effect. Relaxing the error correction threshold, for example by using higher maximum expected error rate or clustering at lower similarities, did not reduce the run effect in replicated samples (Supplementary Fig. S5). Experiment II sequenced in run 2 and run 3 showed a significant run variation in all 24 datasets created using different data processing pipelines (Supplementary Fig. S6). Interestingly, changing quality filtering and clustering methods changed the run and treatment effects in the same direction. Truncating sequences to $180 \mathrm{bp}$ in some cases reduced run variation, possibly due to the merging of some OTUs after truncation, but variation remained relatively large and statistically significant $(P<0.01)$.

Since only three MiSeq runs were used in our study, we are not able to assess the true frequency of sequencing runs that may include biases such as we observed. However, a recent $16 \mathrm{~S}$ study which also reported run-specific biases with taxa-specific effects that were detected using a mock community, observed one such run out of a total of 50 sequencing runs assessed (Yeh et al. 2018). If the incidence of run bias is indeed small, resequencing of biased runs is likely to be a more reliable solution than any correction method. Based on our observations, we have made and archived a large stockpile (enough for more than 10,000 sequencing runs) of a library with unique indices constructed from a master soil sample to serve as technical replicate for all our future sequencing runs. Including the same technical replicate samples in future sequencing runs will be useful for further identifying and estimating the prevalence of outlier runs.

Finally, although it is not clear what the source of bias in run 2 is, we have detected some general features of the vOTUs. For the 24 datasets processed with different error tolerance, the average amplicon length of vOTUs was on average $9.3 \pm 3.3 \%$ shorter than that of other OTUs, and vOTUs had on average $9.7 \pm 2.5 \%$ higher GC content (Supplementary File S4). This contrasts with the observation that amplicon size is in general negatively correlated with PCR and clustering efficiency. Whether such features of OTU variation hold true in other sequencing runs is an interesting and important point for further investigation.

Conclusion. In conclusion, we report an instance of significant variation in microbiome community data among sequencing runs. This variation was not detected using a mock community, run quality metrics, or using less stringent data processing strategies, but was only flagged by examining technical replicates. For large sample sets where sequencing is carried out across multiple sequencing runs, randomized sample assignment, the inclusion of technical replicates, and analysis of run variation should be included to prevent or detect batch effects. Consistent inclusion of technical replicates across sequencing runs will facilitate the detection of critical errors that may be missed by traditional use of mock communities. Even for studies that only use a single sequencing run, including a standard technical replicate can help to identify taxon-specific biases, and help inform downstream bioinformatics processing. It is not clear whether the variation among runs in Ascomycota sequences observed in our dataset will occur in other settings, but the inclusion of biological replicates provides an important first step toward identifying any such systematic variation or bias in sequencing datasets. It is unknown whether specific microbial taxa may be more vulnerable to run variation. While the method we used in detecting and correcting for variable OTUs was tailored for our specific data, it should be possible to develop standardized algorithms for detecting and mitigating run-to-run variation.

\section{ACKNOWLEDGMENTS}

We thank the sequencing support from the University of Minnesota Genomic Center, and computing support from the Minnesota Supercomputing Institute. L. Hanson provided technical support for the project.

\section{LITERATURE CITED}

Al-Ghalith, G. A., Montassier, E., Ward, H. N., and Knights, D. 2016. NINJAOPS: Fast accurate marker gene alignment using concatenated ribosomes. PLOS Comput. Biol. 12:e1004658.

Bengtsson-Palme, J., Ryberg, M., Hartmann, M., Branco, S., Wang, Z., and Godhe, A. 2013. Improved software detection and extraction of ITS1 and ITS2 from ribosomal ITS sequences of fungi and other eukaryotes for analysis of environmental sequencing data. Methods Ecol. Evol. 4:914-919.

Bokulich, N. A., Subramanian, S., Faith, J. J., Gevers, D., Gordon, J. I., Knight, R., et al. 2013. Quality-filtering vastly improves diversity estimates from Illumina amplicon sequencing. Nat. Methods 10:57-59.

Caporaso, J. G., Lauber, C. L., Walters, W. A., Berg-Lyons, D., Huntley, J., Fierer, N., et al. 2012. Ultra-high-throughput microbial community analysis on the Illumina HiSeq and MiSeq platforms. ISME J. 6: 1621-1624

Cole, J. R., Wang, Q., Cardenas, E., Fish, J., Chai, B., Farris, R. J., et al. 2009. The Ribosomal Database Project: Improved alignments and new tools for rRNA analysis. Nucleic Acids Res. 37:D141-D145.

Costea, P. I., Zeller, G., Sunagawa, S., Pelletier, E., Alberti, A., Levenez, F., et al. 2017. Towards standards for human fecal sample processing in metagenomic studies. Nat. Biotechnol. 35:1069-1076.

D’Amore, R., Ijaz, U. Z., Schirmer, M., Kenny, J. G., Gregory, R., Darby, A. C., et al. 2016. A comprehensive benchmarking study of protocols and sequencing platforms for $16 \mathrm{~S}$ rRNA community profiling. BMC Genomics 17:55.

Edgar, R. C., Haas, B. J., Clemente, J. C., Quince, C., and Knight, R. 2011. UCHIME improves sensitivity and speed of chimera detection. Bioinformatics 27:2194-2200.

Essarioui, A., LeBlanc, N., Kistler, H. C., and Kinkel, L. L. 2017. Plant community richness mediates inhibitory interactions and resource competition between Streptomyces and Fusarium populations in the rhizosphere. Microbiol. Ecol. 74:157-167.

Gloor, G. B., Macklaim, J. M., Pawlowsky-Glahn, V., and Egozcue, J. J. 2017. Microbiome datasets are compositional: And this is not optional. Front. Microbiol. 8:2224.

Gohl, D. M., Vangay, P., Garbe, J., MacLean, A., Hauge, A., Becker, A., et al. 2016. Systematic improvement of amplicon marker gene methods for increased accuracy in microbiome studies. Nat. Biotechnol. 34:942-949.

Haas, B. J., Gevers, D., Earl, A. M., Feldgarden, M., Ward, D. V., Giannoukos, G., et al. 2011. Chimeric 16S rRNA sequence formation and detection in Sanger and 454-pyrosequenced PCR amplicons. Genome Res. 21:494-504.

Klindworth, A., Pruesse, E., Schweer, T., Peplies, J., Quast, C., Horn, M., and Glöckner, F. O., 2013. Evaluation of general 16S ribosomal RNA gene PCR primers for classical and next-generation sequencing-based diversity studies. Nucleic Acids Res. 41:1-11

Lindahl, B. D., Nilsson, R. H., Tedersoo, L., Abarenkov, K., Carlsen, T., Pennanen, T., et al. 2013. Fungal community analysis by high throughput sequencing of amplified markers-a user's guide. New Phytol. 199: 288-299.

Nguyen, N. H., Smith, D., Peay, K., and Kennedy, P. 2015. Parsing ecological signal from noise in next generation amplicon sequencing. New Phytol. 205: 1389-1393.

Oksanen, J., Blanchet, F. G., Friendly, M., Kindt, R., Legendre, P., McGlinn, D., et al. 2017. vegan: Community Ecology Package. R package version 2.4-3. https://cran.r-project.org/web/packages/vegan/index.html

Penton, C. R., Gupta, V. V. S. R., Yu, J., and Tiedje, J. M. 2016. Size matters: Assessing optimum soil sample size for fungal and bacterial community structure analyses using high throughput sequencing of rRNA gene amplicons. Front. Microbiol. 7:824.

R Core Team. 2017. R: A language and environment for statistical computing. R Foundation for Statistical Computing, Vienna, Austria. https://www.Rproject.org/

Rognes, T., Flouri, T., Nichols, B., Quince, C., and Mahé, F. 2016. VSEARCH: A versatile open source tool for metagenomics. PeerJ 4:e2584. 
Schloss, P. D., Gevers, D., and Westcott, S. L. 2011. Reducing the effects of PCR amplification and sequencing artifacts on 16S rRNA-based studies. PLoS One 6:e27310.

Smith, D. P., and Peay, K. G. 2014. Sequence depth, not PCR replication, improves ecological inference from next generation DNA sequencing. PLoS One 9:e90234.
Yatsunenko, T., Rey, F. E., Manary, M. J., Trehan, I., Dominguez-Bello, M. G., Contreras, M., et al. 2012. Human gut microbiome viewed across age and geography. Nature 486:222-227.

Yeh, Y.-C., Needham, D. M., and Fuhrman, J. A. 2018. Taxon disappearance from microbiome analysis reinforces the value of mock communities as a standard in every. mSystems 3:e0023-e18. 